\title{
The involvement of working memory and inhibition functions in the different phases of insight problem solving
}

\author{
Kai Lv
}

Published online: 21 January 2015

(C) Psychonomic Society, Inc. 2015

\begin{abstract}
In this article, the involvement of working memory capacity and inhibition functions in different phases of insight problem solving is investigated, by employing a method of separating the different phases of insight problem solving directly, on the basis of the subjects' oral reports. Two experiments are described. In Experiment 1,87 subjects were administered a series of working memory span tasks and inhibition tasks, as well as a verbal insight problem. In Experiment 2, 119 subjects were administered the same working memory span tasks and inhibition tasks as in the first experiment, as well as a spatial insight problem. Several conclusions can be drawn from this study. First, the insight problem-solving process can be divided into several relatively independent phases, including an initial searching phase and a restructuring phase. Second, executive functions, as measured by working memory capacity, influence mainly the initial searching phase, rather than the restructuring phase. Third, inhibition functions play important but complex roles in restructuring, and sometimes could influence restructuring in contradictory ways simultaneously. The implications and value of this study are discussed further.
\end{abstract}

Keywords Insight problem solving $\cdot$ Restructuring $\cdot$ Working memory $\cdot$ Inhibition functions $\cdot$ Phase

\footnotetext{
K. Lv $(\bowtie)$

School of Psychology, Nanjing Normal University, Nanjing, China e-mail: jndxlk2008@sina.com
}

K. Lv

School of Humanity, Jiangnan University, Wuxi, China
Insight problem solving has long concerned many researchers, since Kohler's famous experiments on apes (Kohler, 1925). However, the mechanism underlying insight remains undiscovered. Many investigators believe that insight happens as the result of restructuring, which refers to changing the initial problem representation and forming a new one within which the solution can be found through heuristically guided search processes. There are two conflicting perspectives about the mechanisms of restructuring: One suggests that some automatic and unconscious processes, different from the processes behind analytic problem solving, underlie restructuring (Jung-Beeman et al. 2004; Knoblich et al. 1999; Metcalfe \& Wiebe, 1987; Ohlsson, 1992; Öllinger et al. 2006; Schooler et al. 1993). The other suggests that the processes underlying restructuring are the same as those underlying analytic problem solving (Chronicle et al. 2004; Fleck \& Weisberg, 2004; Gilhooly et al. 2010; Kaplan \& Simon, 1990; Weisberg \& Alba, 1981).

Recently, some researchers have tried to tackle this issue by examining the role of executive functions in insight problem solving. Since executive functions are the crucial predictors in analytic problem solving, evidence about the involvement of executive functions in insight problem solving would provide strong grounds to test different viewpoints on the mechanisms of restructuring. However, the experimental evidence has not been very conclusive. Some investigators have found strong connections between executive functions and insight problem solving, just as in noninsight problem solving (Chein et al. 2010; DeYoung et al. 2008; Gilhooly \& Murphy, 2005; Murray \& Byrne, 2005). However, others have revealed that executive functions played a weaker, or even no, role in insight problem solving (Ash \& Wiley, 2006; Fleck, 2008; Gilhooly \& Fioratou, 2009; Lavric et al. 2000). The inconsistency among the experimental evidence could be due to the 
complexity of insight problem-solving processes, which might include several different phases.

As early as the 1920s, British scholar Wallas (1926) proposed the famous stage theory of the invention and creation processes. This theory suggests that the process of invention or creation could be divided into four stages: the preparation stage, the incubation stage, the illumination stage, and the verification stage. Seifert, Meyer, Davidson, Patalano, and Yaniv (1994) expanded this theory and suggested that each stage could be divided further, into several substages. For example, the preparation stage might include four substages: facing the problem, explaining the failure, storing the failure indices, and stopping solving the problem. Ash and Wiley (2006) also asserted the multiple-stage nature of insight problem solving; they divided the process of insight problem solving into a representation phase, a solution phase, and a restructuring phase.

As can be seen, insight problem solving and noninsight problem solving overlap. For example, in the preparation and solution phases during insight problem solving, the activities of problem solving are similar to those that happen during noninsight problem solving, such as planning, calculating, heuristically guided searching, and so on. The difference is that noninsight problem solving usually reaches success through these activities, whereas insight problem solving does not. This overlap may explain why so many researchers have detected a positive correlation between the performance of insight problem solving and noninsight problem solving (Davidson, 2003; Gilhooly \& Fioratou, 2009; Gilhooly \& Murphy, 2005; Schooler \& Melcher, 1995), and why some investigators have found strong connections between executive functions and insight problem solving, just as in noninsight problem solving. Since the relative importance of different stages of insight problem solving varies with the problem situation, the effects of executive functions on insight problem solving may also vary with the problem situation. This could help explain the inconsistency among the experimental evidence regarding the effects of executive functions on insight problem solving. Nevertheless, due to the multiple-stage nature of such problem solving, the effects of executive functions on the whole insight problem-solving process should not be equal to their effects on the specific phases (e.g., the restructuring phase) of insight problem solving.

In any attempt to uncover the underlying mechanism of restructuring process, the focus should be on the specific phases of insight problem solving rather than on the big picture. Currently, there is still no way to find out when the restructuring process begins during insight problem solving. Nevertheless, it is reasonable to assume that restructuring starts when the initial attempt to solve the problem reaches a state of impasse; that is, the state in which the problem solver feels that he or she has tried every possible way and does not know what to do. The restructuring process will come to an end when the problem solver suddenly realizes the right way to solve the problem, or even the right answer. If we could isolate this phase from the rest during insight problem solving and examine it thoroughly, we might understand the mechanism of the restructuring process better. Ash and Wiley (2006) attempted to do so by comparing the performance of "many moves available" and "few moves available" insight problems. They found that working memory capacity could predict the former but not the latter, which implied that restructuring was unrelated to executive functions.

Although the method of Ash and Wiley (2006) is very ingenious and convincing, it is indirect in examining the effects of executive function on the different phases of insight problem solving. In this study, a method of separating these different phases will be employed, to examine the effects of executive function and other variables on specific phases of insight problem solving. The basic idea of this method is to allow the subjects to report their progress during insight problem solving, which means that the subjects must report both when they feel they are falling into a state of impasse and when they find out the answers. The experimenter recorded these two time points and then determined the durations of the different phases of insight problem solving.

Inhibition functions have been widely explored as one kind of basic mental functions underlying diverse human behaviors, such as perception, attention, memory, language, and so on. However, research into the role of inhibition functions in insight problem solving has been rare. Recently, Storm and colleagues have introduced a few experimental investigations into the effect of inhibition functions on insight problem solving. For example, Storm and Angello (2010) explored the relationship between inhibition functions and performance on the Remote Associates Test (RAT), and found that inhibition functions can be helpful in reducing interference from the irrelevant association words, and thus can improve performance on that test.

Although studies into the role of inhibition functions in insight problem solving have been very rare, the potential relationship between inhibition functions and insight problem solving has been implied in some other domains of investigation, one of which is the connection between inhibition functions and creativity. Eysenck (1993) suggested that high levels of creativity should be related to low levels of inhibition functions such as latent inhibition. This suggestion has been supported by several experiments (Carson et al. 2003; Peterson et al. 2002). However, other studies have suggested a different story. For example, Vartanian (2009) found a significant positive correlation between creativity and the negative-priming effect, which is supposed to increase with the strengthening of inhibition functions. In other words, this study suggested that a high level of creativity was related to a high level of inhibition. Nevertheless, the connection between inhibition functions and creativity seems to exist. Since 
human creativity is highly related to the insight problemsolving process, it is reasonable to assume a potential relationship between inhibition functions and insight problem solving.

Although Storm and Angello (2010) found that inhibition functions could be helpful in solving insight problems such as the RAT, I speculate that the relationship between inhibition functions and insight problem solving should be much more complicated, in that different inhibition functions might have different effects on insight problem solving.

Specifically, spontaneous lateral inhibition function, as measured by the part-set cuing task, should be harmful to restructuring. Spontaneous lateral inhibition happens when one tries to retrieve an item from a group of items belonging to the same category stored in the semantic network, shown in the part-set cuing task. Due to lateral inhibition, the earlyretrieved items will block the retrieval of those items that are not retrieved yet, but important to problem solving. As a result, lateral inhibition function is harmful to the restructuring process. The deleterious effect of lateral inhibition on restructuring could account for the priming effect during insight problem solving. It has been documented that the performance of insight problem solving is damaged if priming stimuli that can reinforce some dominant but inappropriate experience are presented during insight problem solving (Gibson et al. 2011). This phenomenon may be explained by the deleterious effects of lateral inhibition, in that the priming stimuli would enhance lateral inhibition of less dominant but appropriate experience, exerted by the dominant but inappropriate experience.

By contrast, spontaneous self-inhibition function, as measured by the latent inhibition task, should be helpful to restructuring. Spontaneous self-inhibition happens when a stimulus is presented repeatedly without any consequence, as in the latent inhibition task. It would be helpful in suppressing the initially activated but unpromising representations, since the insight problem could not be solved by repetitive searching within the space formed by these representations. As a result, the probability that less dominant but promising representations would be activated would increase, and the restructuring process would become easier.

Voluntary and active inhibition function, as measured by Stroop tasks, should also play an important role in restructuring. I speculate that active inhibition has a negative (i.e., harmful) effect on the restructuring process, because it would suppress representations that are "irrelevant" (but actually crucial) to current problem solving, to ensure that the subject could focus his or her attention on the initially activated representations. As a result, the possibility that those less dominant but crucial representations would be activated would decrease, and restructuring would become more difficult.

Since inhibition functions may play very important but complicated roles in insight problem solving, I will also explore the relationship between inhibition functions and insight problem solving by using the approach mentioned above, as well as multiple tasks, in this study.

It has been suggested in some studies that the type of problem might influence the performance of insight problem solving. For example, Chein et al. (2010) showed that the performance of spatial insight problem solving depended on spatial, but not verbal, working memory capacity. Schooler et al. (1993) found that thinking aloud had different effects on the performance of verbal insight problem solving than on spatial insight problem solving. Gilhooly et al. (2010) also had similar discoveries. In their study, participants who had been given different verbalization instructions attempted different problem types, formed by combining insight versus noninsight and verbal versus spatial factors. The researchers found significant differences between spatial and verbal tasks in terms of both solution rates and latencies. There was also a greater effect of verbalizing on spatial than on verbal problems. Thus, it is possible that the mechanisms of restructuring might be different for verbal and spatial insight problem solving. In this study, I will also compare the processes of verbal and spatial insight problem solving, to investigate the possible role of problem type during insight problem solving.

\section{Experiment 1}

Experiment 1 was designed to investigate the involvement of working memory and inhibition functions in the different phases during verbal insight problem solving. In this experiment, a series of working memory span tasks and inhibition tasks, as well as a verbal insight problem, were administered to the subjects. The method of directly separating the different phases of insight problem solving was employed in order to examine the effects of working memory and inhibition functions on specific phases of verbal insight problem solving.

\section{Method}

\section{Subjects}

The subjects were recruited from Jiangnan University in Wuxi by an advertisement on the campus message board. They were all undergraduates or graduates between the ages of 18 and 25 years. The total number of subjects was 87: 37 men and 50 women.

Tasks

\section{Verbal working memory span tasks}

These tasks were based on those of Conway et al. (2005) and have generally been accepted as measures of verbal working 
memory span, including the reading working memory span task and the operation working memory span task.

Reading working memory span task The purpose of this task was to measure the reading working memory span of the subjects. Seven serials were presented in this task, each composed of two trials with the same number of sentences. In each trial, a group of descriptive sentences were presented to the subjects one by one on the screen of a computer. The subjects had to make a judgment as to whether or not these sentences were grammatical by clicking the "YES" or "NO" button under each sentence with a mouse. At the same time, subjects had to remember the last word of each sentence. When the sentences were all finished, the subjects had to recall the last words of these sentences in the correct order. When the subjects failed in both trials of a serial, the task was terminated. The number of sentences in a trial in a serial started with two, and gradually increased to eight. The highest number of words in a trial that a subject could recall successfully was his or her reading working memory span.

Operation working memory span task The purpose of this task was to measure the operation working memory span of the subjects. The procedure was the same as in the reading working memory span task, except that in this task the sentences were replaced by arithmetic operation equations. The subjects had to make a judgment as to whether or not these equations were correct by clicking the "YES" or "NO" button under each equation, and at the same time they had to remember the word beside each equation. The highest number of words in a trial that a subject could recall successfully was his or her operation working memory span.

\section{Spatial working memory span tasks}

These tasks were based on those of Conway et al. (2005) and are generally accepted as measures of spatial working memory span, including the rotation working memory span task and the symmetry working memory span task.

Rotation working memory span task The purpose of this task was to measure the rotation working memory span of the subjects. The procedure was the same as in the reading working memory span task, except that in this task the materials were different. In each trial, a series of letters with different rotation angles from the standard types or the mirror images of the standard types were presented to the subjects one by one on the screen. The subjects had to make a judgment as to whether or not these letters were formed by rotating standard types, by clicking the "YES" or "NO" button. At the same time, subjects had to remember the direction of an arrow beside each letter. After the letters were all finished, the subjects had to recall the directions of these arrows in the correct order.
In order for the subjects to recall the directions of the arrows, a variety of arrow icons with different directions were presented on the screen, and the subjects had to click those icons with the correct directions in the correct order. The response was scored as correct only when every arrow icon chosen was correct and the order was correct, too. The highest number of arrows in a trial that a subject could recall successfully was his or her rotation working memory span.

Symmetry working memory span task The purpose of this task was to measure the symmetry working memory span of the subjects. The procedure was the same as in the reading working memory span task, except for the materials. In each trial, a series of figures that were symmetric or asymmetric were presented to the subjects one by one on the screen, and the subjects had to make a judgment as to whether or not these figures were symmetric, by clicking the "YES" or "NO" button. At the same time, subjects had to remember the location of a red little square in a big square beside each figure. After the figures were all finished, the subjects had to recall the locations of these red little squares in the correct order. In order for the subjects to recall the locations of the little squares, a variety of little square icons with different locations were presented on the screen, and the subjects had to click those icons with the correct locations in the correct order. The response was scored as correct only when every square icon chosen was correct and the order was correct, too. The highest number of little square locations in a trial that a subject could recall successfully was his or her symmetry working memory span.

In all of these working memory span tasks, higher values of span meant better working memory abilities.

\section{Inhibition tasks}

In this experiment, four tasks were employed to measure the different kinds of inhibition functions. Two Stroop tasks (i.e., the Stroop color word and number tasks) were supposed to tap the active and voluntary inhibition functions. It has been suggested that the performance of Stroop tasks is closely related to the ability to inhibit the interference of irrelevant information and that this kind of inhibition is active and voluntary (Chao, 2011; Logan, 1980; Tzelgov et al. 1992). The latent inhibition task was supposed to tap spontaneous selfinhibition functions. It has been suggested that latent inhibition is some kind of spontaneous self-inhibition, which targets the currently activated stimulus and accumulates with the repetition of the stimulus when no consequences follow (Aguado et al. 1994; Nelson \& del Carmen Sanjuan, 2006; SchmidtHansen et al. 2009). This kind of inhibition happens in a spontaneous and involuntary way. The part-set cuing task was supposed to tap spontaneous lateral inhibition functions. The part-set cuing effect arises from mutual inhibition between exemplars belonging to the same category. This effect 
would increase with the strengthening of lateral inhibition, which is also spontaneous and involuntary (Basden \& Basden, 1995; Foos \& Clark, 2000; Marsh et al. 2004).

Stroop color word task This was a revised version of the task in G. Ward, Roberts, and Phillips (2001). In this task, some stimuli with different colors were presented to the subjects, and the subjects had to identify the color of the stimulus as quickly as possible by pressing the corresponding key (e.g., "S" for red, "K" for green, "X" for blue, and "M" for purple). Two kinds of stimulus were presented in this task. One was color words, including red, green, blue, and purple. The other was character strings, including \#\#\#, $\% \% \%$, and \&\&\&. The task consisted of five blocks, each of which included 36 trials - that is, 12 with color words whose real color was consistent with the meanings of the words, 12 with color words whose real color was inconsistent with their meanings, and 12 with character strings. The presentation sequence of stimuli was randomly assigned. The difference between the average response time (RT) in trials with color words whose real color was inconsistent with their meaning and the average RT in trials with character strings was taken as the index of the Stroop effect.

In this task, higher values of the index of the Stroop effect meant worse inhibition function as measured by this task.

Stroop number task In this task, some stimuli with different sizes were presented to the subjects, and the subjects had to identify the size of the stimulus as quickly as possible by pressing the corresponding key (e.g., "S" to the size of 1 , " $\mathrm{K}$ " to the size of 2 , "X" to the size of 3, and " $\mathrm{M}$ " to the size of 4). There were two kinds of stimulus in this task. One was strings of numbers composed of the same basic number, such as 1, 2, 3, or 4 (e.g., "33," "444"; here, size meant the number of digits in the numbers - e.g., the size of " 444 " was 3 ). The other was character strings that were composed of the same basic character, such as \#, ?, or * (e.g., "\#\#," "???”; here, size meant the number of the characters in a string-e.g., the size of \#\# was 2). The task consisted of five blocks, each of which included 36 trials - that is, 12 with numbers whose size was consistent with the digit of which they were composed (e.g., 333), 12 with numbers whose size was inconsistent with the digit composing the number (e.g., 444), and 12 with character strings. The difference between the average RT in trials with numbers whose size was inconsistent with the constituent digit and the average RT in trials with character strings was taken as the index of the Stroop effect.

In this task, higher values of the index of the Stroop effect meant worse inhibition function as measured by this task.

Latent inhibition task This task was based on SchmidtHansen et al. (2009). There were two stages in this task. In the preexposure stage, six capital letters- $\mathrm{Z}, \mathrm{X}, \mathrm{C}, \mathrm{V}, \mathrm{B}$, and
$\mathrm{N}$-appeared on the screen of a computer, one of which (determined randomly) was then covered by a black square. The subjects had to respond to the covered letter by pressing the corresponding key on the keyboard as quickly as possible after the letter was covered. The order of the six capital letters remained the same in each trial, but the covered letter changed randomly. The background color of the screen during the preexposure stage was always blue, which was manipulated as the preexposure stimulation. In the test stage, some rules were underlying the emergence of the covered letter. The subjects had to find these rules out and predict which letter would be covered by pressing the corresponding key before the letter was covered. When the background color of the screen was blue (i.e., preexposure stimulation), $\mathrm{Z}$ was always covered; when the background color of the screen was green (nonpreexposure stimulation), $\mathrm{N}$ was always covered; when the background color of the screen was red or purple, the letters covered were drawn randomly from $\mathrm{X}, \mathrm{C}, \mathrm{V}$, and B. Due to the latent inhibition effect, the subjects were usually slower in identifying the covered letter corresponding to the preexposure stimulus than in identifying that corresponding to the nonpreexposure stimulation. The difference between the accuracy of responses in the nonpreexposure stimulation trials and the accuracy of response in the preexposure stimulation trials was taken as the index of the latent inhibition effect.

In this task, higher values of the index of the latent inhibition effect meant better inhibition function as measured by this task.

Part-set cuing task This task was based on that of Marsh et al. (2004) and consisted of two stages. In the study stage, the subjects were asked to remember 60 nouns that belonged to four different categories - that is, plant, animal, human, and city. There were 15 nouns in each category. All nouns were presented to the subjects on the computer screen one by one, and the sequence was randomly arranged. In the test stage, the subjects had to recall as many of the nouns as possible. For some categories (i.e., plant and human), only the name of category was provided as the clue to recall; for the other categories (i.e., animal and city), three exemplars belonging to the category were also provided in addition to the name of category, as the clues to recall. Due to the retrieval inhibition effect, it was usually more difficult for the subjects to recall nouns in the categories with exemplars as clues. The difference between the number of nouns correctly recalled in the categories without exemplars and the number of nouns correctly recalled in the categories with exemplars was taken as the index of the part-set cuing effect.

In this task, higher values of the index of the part-set cuing effect meant better inhibition function as measured by this task. 


\section{Insight task}

In this experiment, a verbal insight problem was employed to explore the involvement of working memory and inhibition functions in the different phases during verbal insight problem solving. The problem was a name problem (Weisberg, 1995) and was presented in the following form: "John has travelled around the world without spending a single cent—why?" The answer was that John was some kind of animal, such as a turtle. Most subjects would fall into an impasse when facing this problem, since they always assumed that John was the name of a person. Only when the subjects realized that John was not a human being could this problem be solved. The reason for choosing this problem and the "farm problem" in Experiment 2 as the insight tasks was that pilot studies had shown that the difficulties of these problems were moderate. The goal of the present experiments was to successfully separate the different stages of insight problem solving. On the one hand, the restructuring phase might be too short to be identified and isolated if the insight problem was too easy. On the other hand, the restructuring phase might last too long to end before the time limit of problem solving if the insight problem was too difficult. As a result, the insight problems that were suited to the present study were those that had a moderate degree of difficulty.

The experimenter would first explain the concepts and definitions related to the phases of insight problem solving to the subject and told him or her how to respond. The instructions were as follows

"It has been suggested that the whole process of insight problem solving can usually be divided into several phases. The first phase could be called the initial attempting phase. In this phase, the solvers would try every way to solve the problem. However, most solvers would find that the problem still could not be solved after they had tried every possible way and would feel that they could do nothing about it any more. Thus, the solvers enter into the second phase - that is, the impasse phase. Interestingly, after the solvers have stayed in the state of impasse for some while, the right answer would probably pop into their mind suddenly, without any idea of how this happens. This is the moment of insight. This study is designed to explore the mechanism underlying the process of insight problem solving. Thus, we need you to monitor and report the progress of your own insight problem-solving process. Specifically, when you feel you are entering into the state of impasse, please report to the experimenter; when you think of any answer, please report to the experimenter. However, if you do not experience the feeling of entering into the state of impasse during problem solving, you just need to report the answers to the experimenter."

After the subject had fully understood the instructions, the experimenter presented the insight problem to the subject, and time counting started simultaneously. The maximum time allowed to work on the problem was $50 \mathrm{~min}$. If the subject failed to solve the problem in time, the experimenter would present the right answer. At the end of the experiment, the experimenter interviewed the subjects to learn whether they had encountered this problem or similar problems before.

The insight problem-solving process could be mainly divided into two phases - that is, the initial searching phase and the restructuring phase. The initial searching phase started from the moment of the presentation of the insight problem and ended at the moment of the subject's report of entering the state of impasse. The restructuring phase started from the moment of the subject's report of entering the state of impasse and ended at the moment of the subject's report of the correct answer. Correspondingly, two indices were used to describe the time line of the insight problem-solving process. One was the duration of the initial searching phase (i.e., DIS), which amounted to the interval between the time point of problem presentation and that of the subject's report of entering into impasse. The other was the duration of the restructuring phase (i.e., DR), which amounted to the interval between the time point of the subject's report of entering impasse and that of the subject's report of the correct answer.

In this task, higher values of the two indices meant worse performance in the different phases during insight problem solving.

\section{Procedures}

The study consisted of two separate sessions, and each session lasted about $1 \mathrm{~h}$. The interval between the two sessions was one week. The subjects were randomly assigned to one of two groups, one group finishing all of the working memory span tasks and inhibition tasks in the first session and the insight task in the second session, the other group having the opposite order. Each subject took part in this study individually in both sessions. The working memory span tasks and inhibition tasks were administered on the computer, whereas the insight task was administered on paper.

\section{Results}

The total number of the subjects in this study was 87 , among which two subjects had encountered similar insight problems before and two subjects thought of the right answer shortly after they were presented with the problem and four subjects didn't report entering into the state of impasse. As a result, the number of valid subjects was 79. Among the 79 subjects, 64 solved the insight problem in time. Their performance on all of the tasks is shown in Table 1.

Before further statistical analysis, each of the 64 subjects' performance on all of the tasks was transformed into the standard score. Each subject's standardized spatial working memory span was calculated by averaging the standard scores of 
Table 1 Subjects' performance on the insight task, working memory span tasks, and inhibition tasks in Experiment 1

\begin{tabular}{llll}
\hline & $N$ & Mean & $S D$ \\
\hline DIS & 64 & 192.511 & 164.012 \\
DR & 64 & 892.461 & 864.389 \\
ROTATION & 64 & 1.781 & 0.774 \\
SYMMETRY & 64 & 2.188 & 0.964 \\
OPERATION & 64 & 3.438 & 0.911 \\
READING & 64 & 3.250 & 0.876 \\
LI & 64 & 0.8 & 0.3 \\
PSC & 64 & 6.594 & 2.511 \\
STROOPNUMBER & 64 & 48.956 & 43.598 \\
STROOPCW & 64 & 147.868 & 88.755 \\
\hline
\end{tabular}

DIS, duration of the initial searching phase (in seconds); DR, duration of the restructuring phase (in seconds); ROTATION, Rotation working memory span; SYMMETRY, Symmetry working memory span; OPERATION, Operation working memory span; READING, Reading working memory span; LI, latent inhibition effect (in percentage); PSC, part-set cuing effect; STROOPNUMBER, the Stroop effect on Stroop number task (in milliseconds); STROOPCW, the Stroop effect on Stroop color word task (in milliseconds)

the rotation working memory span and the symmetry working memory span $(r=.56, p<.01)$. Each subject's standardized verbal working memory span was calculated by averaging the standard scores of the operation working memory span and the reading working memory span $(r=.48, p<.01)$. Each subject's standardized Stroop effect was calculated by averaging the standard scores of the Stroop number task and the Stroop color word task $(r=.46, p<.01)$. Then, the correlation matrix was built on the base of the standardized duration of the initial searching phase, the standardized duration of the restructuring phase, the standardized spatial working memory span, the standardized verbal working memory span, the standardized latent inhibition effect, the standardized part-set cuing effect, and the standardized Stroop effect. The results are shown in Table 2.

As is shown in Table 2, no significant correlation emerged between the duration of the initial searching phase and the duration of the restructuring phase. There were significant negative correlations between the duration of the initial searching phase and both spatial and verbal working memory span, as well as a significant positive correlation between the duration of the initial searching phase and the Stroop effect. Significant positive correlations were apparent between the duration of the restructuring phase and the latent inhibition effect, the part-set cuing effect, and the Stroop effect.

Since the correlations between the measures of duration of the different phases of insight problem solving and the measures of inhibition functions might reflect overlapping among the measures of inhibition functions, a hierarchical regression was performed. In this study, the latent inhibition task and the part-set cuing task were supposed to tap spontaneous (involuntary) inhibition functions, whereas the Stroop tasks were supposed to tap active (voluntary) inhibition functions. Accordingly, in the hierarchical regression analysis, with duration of the restructuring phase as the dependent variable, the latent inhibition effect and part-set cuing effect would be entered into the first step, and the Stroop effect in the second step. The results are shown in Table 3. As is shown in this table, after controlling for the spontaneous inhibition measures, the contribution of the active inhibition measures to the duration of the restructuring phase was still significant.

\section{Discussion}

Experiment 1 had several important results. First, there was no significant correlation between the measure of duration of the initial searching phase and the measure of duration of the restructuring phase, which suggested that the initial searching phase and the restructuring phase were relatively independent.

Second, working memory capacity had different effects on the initial searching phase than on the restructuring phase. A significant negative correlation emerged between the measure of the duration of the initial searching phase and the measures of working memory span, which meant that the duration of the initial searching phase decreased as the working memory span increased. However, there was no significant correlation between the duration of the restructuring phase and both measures of working memory span, which suggested that working memory capacity played very little role in the restructuring phase.

Third, a significant positive correlation was apparent between the duration of the initial searching phase and the measure of the Stroop effect. Since the Stroop effect decreased as active inhibition functions increased, this result suggested that active inhibition functions might be helpful to initial searching during verbal insight problem solving.

Fourth, inhibition functions played important roles in the restructuring phase. Significant positive correlations were calculated between the duration of the restructuring phase and several measures of inhibition functions, including the latent inhibition effect, part-set cuing effect, and Stroop effect. These results suggested that the effect of spontaneous inhibition functions on restructuring, as measured by the latent inhibition task and part-set cuing task, was different from the effect of active inhibition functions, as measured by the Stroop tasks. Specifically, spontaneous inhibition functions were harmful to the restructuring process, whereas active inhibition functions were helpful to that process during verbal insight problem solving.

It should be noted that the three inhibition variables were largely uncorrelated with each other. This has been a common finding in the literature, and it had led some to doubt whether there is any unity to the presumed construct of "inhibition." 
Table 2 Correlation matrix of the standardized duration of the initial searching phase (SDIS), duration of the restructuring phase (DR), spatial working memory span (SWM), verbal working memory span (VWM), latent inhibition effect (LI), part-set cuing effect (PSC), and Stroop effect in Experiment 1

\begin{tabular}{lllllll}
\hline & SDIS & SDR & SWM & VWM & SLI & SPSC \\
\hline SDIS & 1.000 & & & & \\
SDR & .209 & 1.000 & & & \\
SWM & $-.564^{* *}$ & .055 & 1.000 & & \\
VWM & $-.273^{*}$ & .112 & .110 & 1.000 & & \\
SLI & .159 & $.354^{* *}$ & .089 & -.001 & 1.000 & 1.000 \\
SPSC & .250 & $.332^{*}$ & $-.298^{*}$ & .085 & .198 & -.130 \\
STROOP & $.415^{* *}$ & $.275^{*}$ & $-.340^{* *}$ & -.127 & -.018 & 1.000 \\
\hline
\end{tabular}

"Inhibition" had been presumed to be one basic construct underlying diverse human mental activities, such as perception, memory, decision making, language, and motion. These activities were so heterogeneous that the inhibition processes underlying them might be quite different. What is more, it was highly possible that there could be different facets in the construct of "inhibition," and that different inhibition tasks tap different facets. As far as this experiment was concerned, the three inhibition tasks (i.e., the Stroop task, latent inhibition task, and part-set cuing task) reflected quite different inhibition functions, which might lead to low correlations among them. Specifically, the Stroop task tapped the voluntary and active inhibition function; the latent inhibition task tapped spontaneous and unconscious self-inhibition; and the part-set cuing task tapped spontaneous lateral inhibition. This issue is still open for discussion.

\section{Experiment 2}

Experiment 2 was designed to investigate the involvement of working memory and inhibition functions in the different phases during spatial insight problem solving. In this experiment, a series of working memory span tasks and inhibition tasks, as well as a spatial insight problem, were administered to the subjects. The method of directly separating the different

Table 3 Hierarchical regression analysis on standardized durations of the restructuring phase in Experiment 1

\begin{tabular}{lllllll}
\hline \multicolumn{2}{l}{ Model } & Beta & $t$ & Sig. & $R^{2}$ & $\Delta \Delta R^{2}$ \\
\hline 1 & LI & .455 & 3.859 & .0001 & & \\
& PSC & .282 & 2.392 & .020 & .371 & \\
2 & LI & .441 & 3.882 & .0001 & & \\
& PSC & .310 & 2.720 & .009 & & \\
& STROOP & .238 & 2.302 & .025 & .527 & .156 \\
\hline
\end{tabular}

LI, latent inhibition effect (as a percentage); PSC, part-set cuing effect phases of insight problem solving was also employed, to examine the effects of working memory and inhibition functions on the specific phases of spatial insight problem solving.

Method

Subjects

The subjects were recruited from Jiangnan University at Wuxi by an advertisement on the campus message board. They were all undergraduates or graduates between the ages of 16 and 25 years. The total number of the subjects was 119: 51 men and 68 women.

\section{Tasks}

Working memory span tasks and inhibition tasks The working memory span tasks included the reading working memory span task, operation working memory span task, rotation working memory span task, and symmetry working memory span task. The inhibition tasks included the Stroop color word task, Stroop number task, latent inhibition task, and part-set cuing task. The materials and procedures of all of these tasks were the same as those employed in Experiment 1.

Insight task In this experiment, a spatial insight problem was employed to explore the involvement of working memory and inhibition functions in the different phases during spatial insight problem solving. The problem was a revised version of the traditional "farm" insight problem (Dow \& Mayer, 2004; see Fig. 1) and was presented in the following form: "There is a farmland with the shape of a ' $\mathrm{V}$ ', as is shown in the figure. How can you divide it into three parts that have the same shape and area?" The answer was to divide the farm into three parts with the shape of a "V."

The experimenter first explained the concepts and definitions of the phases of insight problem solving to the subject and told him or her how to respond. The instructions were the same as those in Experiment 1. After the subject fully understood the 


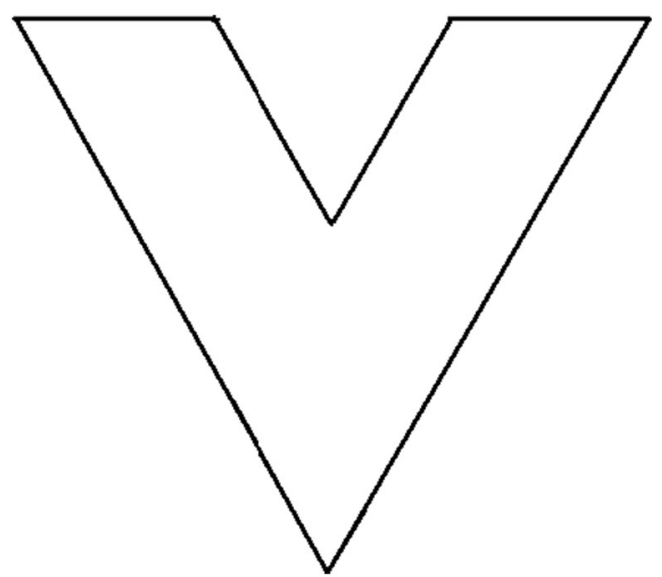

Fig. 1 The "V"-shape farm problem

instructions, the experimenter presented the insight problem to the subject, and time counting started simultaneously. The maximum time allowed to work on the problem was $50 \mathrm{~min}$. If the subject failed to solve the problem in time, the experimenter would present the right answer. At the end of the experiment, the experimenter interviewed the subject to learn whether he or she had encountered this problem or similar problems before. The interval between the time point of problem presentation and the time point of the subject's report of entering into the state of impasse was regarded as the duration of the initial searching phase. The interval between the time points of the subject's report of entering into the state of impasse and the subject's report of the right answer was regarded as the duration of the restructuring phase.

\section{Procedures}

The study consisted of two separate sessions, and each session lasted about $1 \mathrm{~h}$. The interval between the two sessions was one week. The subjects were randomly assigned to one of two groups, with one group finishing all of the working memory span tasks and inhibition tasks in the first session and the insight task in the second session, and the other group having the reverse order. Each subject took part in this study individually in both sessions. The working memory span tasks and inhibition tasks were administered on the computer, whereas the insight task was administered on paper.

\section{Results}

The total number of the subjects in this study was 119 , among whom three thought of the right answer shortly after they were presented with the insight problem, and two did not report entering into the state of impasse. As a result, the number of valid subjects was 114 . Among these 114 subjects, 50 solved the problem in time. Their performance on all of the tasks is shown in Table 4.
Table 4 Subjects' performance on the insight task, working memory span tasks, and inhibition tasks in Experiment 2

\begin{tabular}{llll}
\hline & $N$ & Mean & $S D$ \\
\hline DIS & 50 & 453.667 & 343.956 \\
DR & 50 & $1,640.524$ & $1,118.848$ \\
ROTATION & 50 & 2.010 & 0.723 \\
SYMMETRY & 50 & 2.600 & 1.099 \\
OPERATION & 50 & 3.420 & 0.825 \\
READING & 50 & 3.440 & 0.982 \\
LI & 50 & 5.8 & 2.1 \\
PSC & 50 & 5.208 & 3.155 \\
STROOPNUMBER & 50 & 52.528 & 47.597 \\
STROOPCW & 50 & 159.375 & 87.477 \\
\hline
\end{tabular}

DIS, duration of the initial searching phase (in seconds); DR, duration of the restructuring phase (in seconds); ROTATION, rotation working memory span; SYMMETRY, symmetry working memory span; OPERATION, operation working memory span; READING, reading working memory span; LI, latent inhibition effect (as a percentage); PSC, partset cuing effect; STROOPNUMBER, Stroop effect on the Stroop number task (in milliseconds); STROOPCW, Stroop effect on the Stroop color word task (in milliseconds)

Before further statistical analysis, each of the 50 subjects' performance on all of the tasks was transformed into a standard score. Each subject's standardized spatial working memory span was calculated by averaging the standard scores of rotation working memory span and symmetry working memory $\operatorname{span}(r=.55, p<.01)$. Each subject's standardized verbal working memory span was calculated by averaging the standard scores of operation working memory span and reading working memory $\operatorname{span}(r=.61, p<.01)$. Each subject's standardized Stroop effect was calculated by averaging the standard scores of the Stroop number task and the Stroop color word task $(r=.51, p<.01)$. Then, the correlation matrix was built on the base of the standardized duration of the initial searching phase, standardized duration of the restructuring phase, standardized spatial working memory span, standardized verbal working memory span, standardized latent inhibition effect, standardized part-set cuing effect, and standardized Stroop effect. The results are shown in Table 5.

As is shown in Table 5, no significant correlation was apparent between the duration of the initial searching phase and the duration of the restructuring phase. A significant negative correlation emerged between the duration of the initial searching phase and spatial working memory span. There was a significant positive correlation between the duration of the initial searching phase and the Stroop effect. As far as the duration of the restructuring phase was concerned, only one correlation coefficient reached a significant level-that is, a negative correlation between the duration of the restructuring phase and the latent inhibition effect. 
Table 5 Correlation matrix of the standardized duration of the initial searching phase (SDIS), duration of the restructuring phase (SDR), spatial working memory span (SWM), verbal working memory span (VWM), latent inhibition effect (SLI), part-set cuing effect (SPSC), and Stroop effect in Experiment 2

\begin{tabular}{|c|c|c|c|c|c|c|c|}
\hline & SDIS & SDR & SWM & VWM & SLI & SPSC & STROOP \\
\hline SDIS & 1.000 & & & & & & \\
\hline SDR & -.077 & 1.000 & & & & & \\
\hline SWM & $-.277^{*}$ & .231 & 1.000 & & & & \\
\hline VWM & -.209 & .153 & $.625^{* *}$ & 1.000 & & & \\
\hline SLI & -.151 & $-.282^{*}$ & .168 & .108 & 1.000 & & \\
\hline SPSC & -.105 & .101 & -.257 & -.040 & -.128 & 1.000 & \\
\hline STROOP & $.365^{*}$ & -.009 & -.145 & .019 & .226 & -.063 & 1.000 \\
\hline
\end{tabular}

\section{Discussion}

Several important results emerged from Experiment 2. First, there was no significant correlation between the durations of the initial searching phase and the restructuring phase, which replicated the results of Experiment 1 and supported the assertion that the initial searching phase and the restructuring phase are relatively independent.

Second, working memory capacity had different effects on the initial searching phase and the restructuring phase. There were negative correlations between the duration of the initial searching phase and both measures of working memory span, although the correlation coefficient between the duration of the initial searching phase and the measure of verbal working memory span did not reach a significant level. What is more, no correlation was significant between the duration of the restructuring phase and the two measures of working memory span. Again, these results basically replicated those of Experiment 1 and suggested that working memory capacity plays a greater role in the initial searching phase than in the restructuring phase.

Third, a significant positive correlation emerged between the duration of the initial searching phase and the Stroop effect. This result also replicated the results of Experiment 1 and suggested that active inhibition functions might be helpful to the initial searching during insight problem solving.

Fourth, inhibition functions played a role in the restructuring phase. There was a significant negative correlation between the duration of the restructuring phase and the latent inhibition effect, which suggested that the spontaneous inhibition functions measured by the latent inhibition task are helpful to the restructuring process. This result was opposite to the result in Experiment 1, in which the spontaneous inhibition functions were harmful to the restructuring process. In addition, no significant correlation was found between the duration of the restructuring phase and the other two measures of inhibition functions.

\section{General discussion}

Although the underlying mechanism of insight problem solving remains a secret, many investigators believe that insight problem-solving process can be divided into several relatively independent phases (Ash \& Wiley, 2006; Seifert et al. 1994; Wallas, 1926). However, little research has explored the multiphase nature of insight problem solving directly. In this study, a method of separating the different phases of insight problem solving was employed to examine the effects of executive functions and inhibition functions on the specific phases of insight problem solving. Several interesting results were revealed by this study.

First of all, the initial searching phase and the restructuring phase were relatively independent. There was no significant correlation between the duration of the initial searching phase and that of the restructuring phase in either verbal or spatial insight problem solving. This result not only supported the argument that the insight problem-solving process can be divided into several relatively independent phases, but also suggested that the method to separate the different phases of insight problem solving employed in this study was somewhat effective.

Another interesting result was that working memory capacity had different effects on the initial searching phase and the restructuring phase. In both verbal and spatial insight problem solving, working memory capacity was helpful to the initial searching, but irrelevant to restructuring. In other words, executive function, as measured by working memory span, exerted its influence mainly on the initial searching rather than on the restructuring process. This result favors the "specialprocesses" view (Jung-Beeman et al. 2004; Knoblich et al. 1999; Metcalfe \& Wiebe, 1987; Ohlsson, 1992; Öllinger et al. 2006, Öllinger et al. 2008; Schooler et al. 1993) against the "business-as-usual" view (Chronicle et al. 2004; Fleck \& Weisberg, 2004; Gilhooly et al. 2010; Kaplan \& Simon, 1990; Weisberg \& Alba, 1981), regarding the nature of restructuring. 
Perhaps the most interesting result of this study was that inhibition functions played important but complex roles in restructuring. These results were very interesting and intriguing.

In insight problem solving in both experiments, the effects of latent inhibition on restructuring were significant. However, the effect was negative in verbal insight problem solving, but positive in spatial insight problem solving. The hypothesis that spontaneous self-inhibition function, as measured by the latent inhibition task, should be helpful to restructuring was only partially supported (i.e., in spatial insight problem solving). In verbal insight problem solving, the effect of selfinhibition function on restructuring was reversed. One explanation for this paradoxical result is that spontaneous selfinhibition might play a very complex role in the restructuring process - that is, it might be both helpful and harmful to restructuring, simultaneously. On the one hand, it could suppress those initially activated experiences or representations, which are "relevant" or "strong" but are actually unhelpful to solving the problem after continuous failure. Thus, space is opened for those "irrelevant" or "weak" experiences or representations that are actually vital to solving the problem. On the other hand, spontaneous self-inhibition might reflect some kind of inherent inhibition ability of a person, which could target all representations, including those that are necessary to restructuring. As a result, the actual effect of the inhibition function on restructuring would be the combination of these two contradictory effects, which may be mediated by many factors, such as problem type, situation, time course, and so on. This might explain the different patterns of effects of inhibition function across the different insight problems.

The hypothesis that spontaneous lateral inhibition function, as measured by the part-set cuing task, should be harmful to restructuring was supported in the verbal insight task. However, the effect of lateral inhibition function on restructuring was not significant in the spatial insight task, although the correlation coefficient suggested the same trend as in the verbal insight task.

The hypothesis that active inhibition, as measured by the Stroop tasks, has a negative effect on the restructuring process was not supported in the verbal insight task; on the contrary, active inhibition facilitated restructuring significantly in the verbal insight problem solving. In the spatial insight task, the effect of active inhibition on restructuring was not significant, although the correlation coefficient suggested an opposite trend from that in the verbal insight task. According to these results, it is reasonable to assume that active inhibition might be both helpful and harmful to restructuring simultaneously, just as spontaneous self-inhibition may be. On the one hand, active inhibition could suppress those representations that are regarded as "irrelevant" but are actually important to problem solving, to make sure that at first the subject can focus his or her attention on the initially activated representations. As a result, the possibility that those weaker but important representations would be activated would decrease, and restructuring would become more difficult. This "help focus attention" function of active inhibition was supported by the relationship between the Stroop effect and the initial searching phase. In both verbal and spatial insight problem solving, significant positive correlations were apparent between the Stroop effect and initial search duration, which suggested that active inhibition facilitated the initial search. On the other hand, active inhibition may also suppress those initially activated but inappropriate representations after the subject continues to experience failure during insight problem solving. As a result, the possibility that those weaker but important representations would be activated would increase, and restructuring would become easier. The actual effect of active inhibition on restructuring should be the combination of these two contradictory effects, which might be mediated by problem type or some other factors.

Few studies have explored the role of inhibition functions during insight problem solving, especially during the restructuring process (Storm \& Angello, 2010). Thus, this study may provide some valuable perspective into this issue. As this study has suggested, the effects of inhibition functions on insight problem solving, especially the restructuring phase, are very complicated and sometimes contradictory. Different inhibition functions have different effects on restructuring. What is more, some inhibition functions might influence restructuring in the contradictory ways simultaneously. I believe that the nature of these complex effects of inhibition functions on restructuring will be worthy of further investigation.

Since it has been suggested that the type of insight problems may influence the performance of insight problem solving (Chein et al. 2010; Gilhooly et al. 2010; Schooler et al. 1993), I also compared the effects of working memory capacity and inhibition functions on verbal versus spatial insight problem solving. The results showed many similarities between these two types of insight problem solving. For example, in both types of insight problem solving, the initial search phase and the restructuring phase were relatively independent, and working memory capacity was helpful to initial searching, but irrelevant to restructuring, and inhibition functions played important roles in restructuring.

However, there are some differences in the patterns of inhibition effects on the restructuring. For example, the effect of spontaneous self-inhibition on restructuring in verbal insight problem solving was contrary to that in spatial insight problem solving. The effects of spontaneous lateral inhibition and active inhibition on restructuring were significant in verbal insight problem solving, but not significant in spatial insight problem solving. One explanation for these differences is that many inhibition functions, such as spontaneous self-inhibition and active inhibition, could influence restructuring in contradictory ways simultaneously. The actual effect of a specific inhibition function on restructuring would be the combination of the two contradictory effects, which could be mediated by many 
factors, such as problem type, situation, time course, and so on. Another possibility is that there may be complex interactions among the different inhibition functions, which may vary with the problem situation. This issue is open for investigation.

It should be pointed out that there may be some methodological weaknesses in this study, which may limit the generalizability of the findings. One is related to the reliability of subjective reports. Although most subjects can realize and distinguish between the different states during insight problem solving, such as impasse and insight, the accuracy of their reports on the time points of different stages remains a question. For example, the reports on the time point of entering the impasse stage might precede or fall behind the real time point of entering impasse. Another problem is the subjects' readiness to respond. It has been revealed in this study that some subjects are very reluctant to report their states during insight problem solving. For example, they did not report in time when they had insights about the problem solution. The reason might be that they were not sure whether their insights were correct. There may be a few possible ways to remedy these weaknesses in future study. For example, a thorough verbal protocol for subjects may be helpful in identifying the time points of the different stages of insight problem solving more accurately, along with subjects' time reports. Subjects should be encouraged to respond more actively, without worrying about the correctness of their responses. In addition, some neurophysiological approaches could also be used to separate the stages, such as eye movement tracking, electroencephalography (EEG), and functional magnetic resonance imaging (fMRI). These methods have proved to be very powerful in revealing some of the underlying processes that subjects could not realize or report precisely. For example, Kounios et al. (2006) revealed that insight was associated with less alpha power in midfrontal cortex and left anterior temporal cortex. Jung-Beeman et al. (2004) found that gamma activity increased shortly before insight response at anterior right temporal areas. These studies suggested some connections between neurophysiological activity and the processes of insight problem solving. I believe that with the help of neurophysiological approaches, we could separate the different phases of insight problem solving more precisely.

\section{Conclusion and prospection}

Several conclusions can be drawn from this study. First, insight problem-solving processes can be divided into several relatively independent phases, such as the initial searching phase and the restructuring phase. Second, executive function as measured by working memory capacity exerts its influence mainly on the initial searching phase rather than on the restructuring phase, which favors the "special-processes" view against the "business-as-usual" view regarding the nature of restructuring. Third, inhibition functions play important but complex roles in restructuring: Spontaneous lateral inhibition is harmful to restructuring. Spontaneous selfinhibition and active inhibition, on the other hand, could both influence restructuring in contradictory ways simultaneously, so that the actual effects on restructuring would be the combination of the two contradictory effects, which might vary with the problem situations.

It should be stated that several issues in this study are worthy of examination in future research. The first is how to separate the different phases of the insight problem-solving process more effectively. The method employed in this study is tentative, and its effectiveness remains to be confirmed. Due to the uncertainty and subjectivity of oral report, some more objective methods should be developed in the future. For example, some neurophysiological approaches might be helpful in separating the different stages of insight problem solving, such as eye movement tracking, EEG, fMRI, and so on. The second issue worthy of further study is the choice of inhibition tasks. In this study, I employed Stroop tasks as the measure of active inhibition function, the latent inhibition task as the measure of spontaneous self-inhibition function, and the part-set cuing task as the measure of spontaneous lateral inhibition function. However, there are some disagreements as to the natures of these tasks. For example, some researchers have asserted that latent inhibition arises because of the formation of a connection between the conditioned stimulus and the occurrence of a "nonevent," instead of through the effect of real inhibition functions (Hall, 1991; Hall \& Rodriguez, 2011). Therefore, to confirm the effects of inhibition functions on insight problem solving, other inhibition tasks should be employed. The third aspect I wish to highlight is the nature of the effects of inhibition functions on restructuring. As this study has shown, the effects of inhibition functions on restructuring are very complex, and even contradictory sometimes. Why and how does this happen? Are there interactions among these effects? How could we separate the opposite effects of a specific inhibition function on restructuring? All of these questions remain to be answered. Insight problem solving, especially the mechanism of insight, may be one of the most interesting topics for psychological investigation. By means of comparing the effects of multiple variables on different phases during insight problem solving, we may be able to understand this phenomenon better.

\section{References}

Aguado, L., Symonds, M., \& Hall, G. (1994). Interval between preexposure and test determines the magnitude of latent inhibition: Implications for an interference account. Animal Learning \& Behavior, 22, 188-194. doi:10.3758/BF03199919 
Ash, I. K., \& Wiley, J. (2006). The nature of restructuring in insight: An individual-differences approach. Psychonomic Bulletin \& Review, 13, 66-73. doi:10.3758/BF03193814

Basden, D. R., \& Basden, B. H. (1995). Some tests of the strategy disruption interpretation of part-list cuing inhibition. Journal of Experimental Psychology. Learning, Memory, and Cognition, 21, 1656-1669. doi:10.1037/0278-7393.21.6.1656

Carson, S. H., Peterson, J. B., \& Higgins, D. M. (2003). Decreased latent inhibition is associated with increased creative achievement in highfunctioning individuals. Journal of Personality and Social Psychology, 85, 499-506.

Chao, H.-F. (2011). Active inhibition of a distractor word: the distractor precue benefit in the Stroop color-naming task. Journal of Experimental Psychology. Human Perception and Performance, 37, 799-812.

Chein, J. M., Weisberg, R. W., Streeter, N. L., \& Kwok, S. (2010). Working memory and insight in the nine-dot problem. Memory \& Cognition, 38, 883-892. doi:10.3758/MC.38.7.883

Chronicle, E. P., MacGregor, J. N., \& Ormerod, T. C. (2004). What makes an insight problem? The roles of heuristics, goal conception, and solution recoding in knowledge-lean problems. Journal of Experimental Psychology. Learning, Memory, and Cognition, 30, 14-27. doi:10.1037/0278-7393.30.1.14

Conway, A. R. A., Kane, M. J., Bunting, M. F., Hambrick, D. Z., Wilhelm, O., \& Engle, R. W. (2005). Working memory span tasks: A methodological review and user's guide. Psychonomic Bulletin \& Review, 12, 769-786. doi:10.3758/BF03196772

Davidson, J. E. (2003). Insights about insightful problem solving. In J. E. Davidson \& R. J. Sternberg (Eds.), The psychology of problem solving (pp. 149-175). New York, NY: Cambridge University Press.

DeYoung, C. G., Flanders, J. L., \& Peterson, J. B. (2008). Cognitive abilities involved in insight problem solving: an individual differences model. Creativity Research Journal, 20, 278-290.

Dow, G. T., \& Mayer, R. E. (2004). Teaching students to solve insight problems: Evidence for domain specificity in creativity training. Creativity Research Journal, 16, 389-402.

Eysenck, H. J. (1993). Creativity and personality: Suggestions for a theory. Psychological Inquiry, 4, 147-178.

Fleck, J. I. (2008). Working memory demands in insight versus analytic problem solving. European Journal of Cognitive Psychology, 20, $139-176$.

Fleck, J. I., \& Weisberg, R. W. (2004). The use of verbal protocols as data: An analysis of insight in the candle problem. Memory \& Cognition, 32, 990-1006. doi:10.3758/BF03196876

Foos, P. W., \& Clark, M. C. (2000). Old age, inhibition, and the part-set cuing effect. Educational Gerontology, 26, 155-160.

Gibson, J. M., Dhuse, S., Hrachovec, L., \& Grimm, L. R. (2011). Priming insight in groups: Facilitating and inhibiting solving an ambiguously worded insight problem. Memory \& Cognition, 39, 128-146. doi: 10.3758/s13421-010-0014-7

Gilhooly, K. J., \& Fioratou, E. (2009). Executive functions in insight versus non-insight problem solving: An individual differences approach. Thinking \& Reasoning, 15, 355-376.

Gilhooly, K. J., Fioratou, E., \& Henretty, N. (2010). Verbalization and problem solving: Insight and spatial factors. British Journal of Psychology, 101, 81-93.

Gilhooly, K. J., \& Murphy, P. (2005). Differentiating insight from noninsight problems. Thinking \& Reasoning, 11, 279-302.

Hall, G. (1991). Perceptual and associative learning. Oxford, UK: Oxford University Press, Clarendon Press.

Hall, G., \& Rodriguez, G. (2011). Blocking of potentiation of latent inhibition. Journal of Experimental Psychology: Animal Behavior Processes, 37, 127-131.

Jung-Beeman, M., Bowden, E. M., Haberman, J., Frymiare, J. L., Arambel-Liu, S., Greenblatt, R., et al. (2004). Neural activity when people solve verbal problems with insight. PLoS Biology, 2(E97), 500-510. doi:10.1371/journal.pbio.0020097

Kaplan, G. A., \& Simon, H. A. (1990). In search of insight. Cognitive Psychology, 22, 374-419.

Knoblich, G., Ohlsson, S., Haider, H., \& Rhenius, D. (1999). Constraint relaxation and chunk decomposition in insight problem solving. Journal of Experimental Psychology. Learning, Memory, and Cognition, 25, 1534-1555. doi:10.1037/0278-7393.25.6.1534

Kohler, W. (1925). The mentality of apes. London: Routledge.

Kounios, J., Frymiare, J. L., Bowden, E. M., Fleck, J. I., Subramaniam, K., Parrish, T. B., et al. (2006). The prepared mind: Neural activity prior to problem presentation predicts subsequent solution by sudden insight. Psychological Science, 17, 882-890. doi:10.1111/j. 1467-9280.2006.01798.x

Lavric, A., Forstmeier, S., \& Rippon, G. (2000). Differences in working memory involvement in analytical and creative tasks: An ERP study. Cognitive Neuroscience, 11, 1613-1618.

Logan, G. D. (1980). Attention and automaticity in Stroop and priming tasks: Theory and data. Cognitive Psychology, 12, 523-553.

Marsh, E. J., Dolan, P. O., Balota, D. A., \& Roediger, H. L. (2004). Partset cuing effects in younger and older adults. Psychology and Aging, 19, 134-144. doi:10.1037/0882-7974.19.1.134

Metcalfe, J., \& Wiebe, D. (1987). Intuition in insight and non-insight problem solving. Memory \& Cognition, 15, 238-246.

Murray, M. A., \& Byrne, R. M. J. (2005). Attention and working memory in insight problem solving. In B. G. Bara, L. Barsalou, \& M. Bucciarelli (Eds.), Proceedings of the 27th Annual Conference of the Cognitive Science Society (pp. 1571-1575). Mahwah, NJ: Erlbaum.

Nelson, J. B., \& del Carmen Sanjuan, M. (2006). A context-specific latent inhibition effect in a human conditioned suppression task. Quarterly Journal of Experimental Psychology, 59, 1003-1020. doi:10.1080/ 17470210500417738

Ohlsson, S. (1992). Information-processing explanations of insight and related phenomena. In M. Keane \& K. Gilhooly (Eds.), Advances in the psychology of thinking (pp. 1-44). London, UK: HarvesterWheatsheaf.

Öllinger, M., Jones, G., \& Knoblich, G. (2006). Heuristics and representational change in two move matchstick arithmetic tasks. Advances in Cognitive Psychology, 2, 239-253.

Öllinger, M., Jones, G., \& Knoblich, G. (2008). Investigating the effect of mental set on insight problem solving. Experimental Psychology, $55,269-282$

Peterson, J. B., Smith, K. W., \& Carson, S. (2002). Openness and extraversion are associated with reduced latent inhibition: Replication and commentary. Personality and Individual Differences, 33, 1137-1147.

Schmidt-Hansen, M., Killcross, A. S., \& Honey, R. C. (2009). Latent inhibition, learned irrelevance, and schizotypy: Assessing their relationship. Cognitive Neuropsychiatry, 14, 11-29.

Schooler, J. W., \& Melcher, J. (1995). The ineffability of insight. In S. M. Smith, T. B. Ward, \& R. A. Finke (Eds.), The creative cognition approach (pp. 97-143). Cambridge, MA: MIT Press.

Schooler, J. W., Ohlsson, S., \& Brooks, K. (1993). Thoughts beyond words: When language overshadows insight. Journal of Experimental Psychology: General, 122, 166-183. doi:10.1037/ 0096-3445.122.2.166

Seifert, C. M., Meyer, D. E., Davidson, N., Patalano, A. L., \& Yaniv, I. (1994). Demystification of cognitive insight: Opportunistic assimilation and the prepared-mind hypothesis. In R. Sternberg \& J. Davidson (Eds.), The nature of insight (pp. 65-124). Cambridge, MA: MIT Press.

Storm, B. C., \& Angello, G. (2010). Overcoming fixation: Creative problem solving and retrieval-induced forgetting. Psychological Science, 21, 1263-1265. doi:10.1177/0956797610379864

Tzelgov, J., Henik, A., \& Berger, J. (1992). Controlling Stroop effects by manipulating expectations for color words. 
Memory \& Cognition, 20, 727-735. doi:10.3758/BF0320 2722

Vartanian, O. (2009). Variable attention facilitates creative problem solving. Psychology of Aesthetics, Creativity, and the Arts, 3, 57-59.

Wallas, G. (1926). Art of thought. New York, NY: Harcourt, Brace and Co.

Ward, G., Roberts, M. J., \& Phillips, L. H. (2001). Task switching costs, Stroop costs, and executive control: A correlational study. Quarterly Journal of Experimental Psychology, 54A, 491-511.
Weisberg, R. W. (1995). Prolegomena to theories of insight in problem solving: A taxonomy of problems. In R. J. Sternberg \& J. E. Davidson (Eds.), The nature of insight (pp. 157-196). Cambridge, MA: MIT Press.

Weisberg, R. W., \& Alba, J. W. (1981). An examination of the alleged role of "fixation" in the solution of several "insight" problems. Journal of Experimental Psychology: General, 110, 169-192. doi: 10.1037/0096-3445.110.2.169 